\title{
Seroprevalence against classical swine fever virus in vaccinated pigs in Ho Chi Minh City
}

\author{
Ha T. N. $\operatorname{Tran}^{1}$, Phuong P. B. Nguyen ${ }^{2}$, Thanh D. H. Le ${ }^{2}$, Hien T. Le ${ }^{1}$, \& Mai C. Duong ${ }^{1 *}$ \\ ${ }^{1}$ Faculty of Animal Science and Veterinary Medicine, Nong Lam University, Ho Chi Minh City, Vietnam \\ ${ }^{2}$ Division of Sub-department of Animal Health Ho Chi Minh City, Vietnam
}

ARTICLE INFO
Research Paper
Received: March 30, 2020
Revised: May 20, 2020
Accepted: June 22, 2020
Keywords
Antibody
Antigen
CSF
ELISA
Swine
*Corresponding author
Duong Chi Mai
Email: mai.duongchi@hcmuaf.edu.vn
F

\begin{abstract}
The aim of this study was to survey the serological response to classical swine fever disease in vaccinated pigs in $\mathrm{Cu} \mathrm{Chi}$, Ho Chi Minh City. By using the PrioCHECK ${ }^{\circledR}$ CSFV Ab 2.0 ELISA test kit to detect antibodies against CSF in 410 vaccinated pigs and IDEXX CSFV Ag Serum Plus Test to detect the $\mathrm{E}^{\mathrm{rns}}$ protein of the CSFV in pigs without antibodies against CSFV. Results showed that the overall seroprevalence observed in vaccinated pigs in other Farms varied from $70 \%-100 \%(P<0.05)$, but in Farm 5, no pigs produced a positive humoral response against CSFV were found. The highest seroprevalence of antibodies against CSFV was found in Farms with a herd size of $\geq 1000-<6000$ animals $(91.26 \%)$ and the lowest was a Farms with less than 1000 animals (51.81\%). The highest ratio of positive pigs for antibodies against CSFV belonging to Group of $>40-\leq 60$ days post-vaccination was $98.36 \%$; and the lowest rate was found in Group of $30-\leq 40$ days postvaccination $(51.96 \%)$. That grower pigs had the highest proportion of positive pigs for antibodies against CSFV accounting for $81.40 \%$; next, the proportion of positive sows was $73.24 \%$. Significant differences in the seroprevalence observed in vaccinated pigs across herd size, days post-vaccination, type of pigs $(P<0.05)$. In this study, no pig was found to contain CSFV antigen.
\end{abstract}

Cited as: Tran, H. T. N., Nguyen, P. P. B., Le, T. D. H., Le, H. T., \& Duong, M. C. (2020). Seroprevalence against classical swine fever virus in vaccinated pigs in Ho Chi Minh City. The Journal of Agriculture and Development 19(3), 48-51.

\section{Introduction}

Classical swine fever (CSF) also known as hog cholera, is one of the most important viral diseases of domestic pigs and wild boar. In infected pigs, the CSF virus causes one of several forms (acute, chronic, or prenatal) and can result in high morbidity and mortality in swine. In 1997, an outbreak of CSF occurred in the Netherlands and caused more than $€ 2.5$ billion (EURO) in losses and more than 11 million pigs were destroyed (Meuwissen et al., 1999). CSF disease still exists and causes economic losses to the swine industry in many countries in Asia, Latin America and Europe (WAHID, 2015). Strategies to control classical swine fever virus (CSFV) mainly consist of stamping out policy (non-vaccination) and a systematic prophylactic vaccination (Huang et al., 2014). In Vietnam, since 1980, with systemic prophylactic vaccination, severe CSF outbreaks have been controlled in pig farms. Therefore, the development of management programs, proactive vaccination, vaccination control, post-vaccination surveillance for CSF disease is essential to reduce the damage caused by the disease. Especially in Ho Chi Minh City, the CSF vaccine has been recommended in the routine vaccination program for animals according to Decision no. 07/2016/TT-BNNPTNT about the national program of controlling CSF disease issued in 2016 
(MARD, 2016). For that reason, the purpose of this study is to assess the serological response to classical swine fever disease in vaccinated pigs in $\mathrm{Cu}$ Chi, Ho Chi Minh City.

\section{Materials and Methods}

Total 410 pigs from 7 pig farms in 6 wards (An Nhon Tay, Hoa Phu, Nhuan Duc, Pham Van Coi, Phu My Hung, Trung Lap Thuong) of $\mathrm{Cu}$ Chi district, Ho Chi Minh city were selected at random and approved for use in this study by Animal Health Laboratory and Treatment Division, Subdepartment of Animal Health and Husbandry of Ho Chi Minh City. A total of 410 serum samples were originally collected as part of on-going annual disease investigations. In each farm, depending on herd size and the permission of owners, 5 100 pigs were collected randomly for blood sampling. All pigs were vaccinated Coglapest (Ceva, Hungary) or Dich Ta Heo (Navetco, Vietnam) against CSFV and blood samples were collected from 30 to more than 60 days post-vaccination. All serum samples were tested for the presence of antibody against CSFV; then, a total of 108 serum samples were negative for CSF antibodies would be detected $\mathrm{E}^{\mathrm{rns}}$ antigen by using IDEXX CSFV Ag Serum Plus Test kit. PrioCHECK ${ }^{\prime \prime}$ CSFV Ab 2.0 (Prionics Lelystad B.V. Netherlands) is used to detect antibodies against CSFV and IDEXX CSFV Ag Serum Plus Test is used for the presence of $\mathrm{E}^{\mathrm{rns}}$ protein of CSFV. The ELISA procedures of this study were performed according to the manufacturer's recommendations. Descriptive analysis was performed and reported as a mean value. Chi-square test was used to compare the difference in proportions of seroprevalence of age groups, breeds, and other variables. The difference level $P<0.05$ will be considered as significant difference.

\section{Results and Discussion}

The overall seroprevalence observed in vaccinated pigs was $73.66 \%(302 / 410)$. Compared with previous studies, the higher seroprevalences of vaccinated pigs in the study by Lam Hoang Kiet but lower seroprevalences of vaccinated pigs in the study by Nguyen Le Thanh were 65,63\% and $75.66 \%$, respectively (Lam, 2009; Nguyen, 2018). In this study, no pig was positive for the presence of antibodies against CSFV in Farm 5 was found. There were significant differences in the seroprevalence observed in vaccinated pigs across Farms $(P<0.05)$. In this study, except Farm 5, six other Farms met the demand of the Sub-department of Animal Health Ho Chi Minh scheme (MARD, 2016), which required the ratio of a positive result against CSFV antibody of a herd must be greater than $70 \%$. According to Blome et al. (2017), CSF vaccination was still in use to reduce the disease burden in endemically affected countries. As mentioned above, all pigs vaccinated Coglapest (Ceva, Hungary) or Dich Ta Heo (Navetco, Vietnam) against CSFV were selected randomly for the detection the seroprevalence of pigs from CSFV; therefore, it is hard to explain why Farm 5 had no positive pigs for antibodies against CSFV. A previous study suggested that antibody responses against the CSF vaccine were significantly reduced in Trypanosoma evansi infected pigs as compared to uninfected pigs. This immunosuppression might explain the accounts of poor protection of CSF vaccinated pigs reported in T. evansi endemic areas of Vietnam (Holland et al., 2003). It is likely that poor handling and malpractice in CSF vaccination in Farm 5 occurred and this leads to the failure in CSF vaccination on this Farm. In reality, the owners were responsible for vaccination programs in a pig farm and the information about these pigs such as vaccination programs was collected by using questionnaire lists. Furthermore, maternally derived antibody was the most common cause of CSFV vaccination failure, particularly in highly endemic areas (Suradhat et al., 2007), and therefore piglets that have circulating maternal antibody may not seroconvert when vaccinated.

A significant difference in positive pigs among the three groups of herd sizes was found $(P<$ 0.05) in Table 1. According to Moening (2000) and Guo et al. (2011), the percentage of positive pigs for antibodies against CSFV influenced by many factors, such as the type of antigen in the vaccine, the integrity of the vaccine, age of pigs when vaccinated (associated with maternal antibody), application of vaccination by officers, environmental conditions and pig health condition. The success of vaccination programs is determined by the formation of protective antibodies in pigs (Ratundima et al., 2012). It is likely that Farms with herd sizes over 1000 animals applied proper hygienic and disease prevention procedures.

Based on days post-vaccination, there was a significant difference about the seroprevalence 
Table 1. The seroprevalence of antibodies against classical swine fever virus

\begin{tabular}{ccccc}
\hline & & Number of samples & $\begin{array}{c}\text { Number of positive } \\
\text { samples }\end{array}$ & Ratio (\%) \\
\hline \multirow{5}{*}{ Farm } & 1 & 61 & 60 & 98.36 \\
& 2 & 61 & 61 & 100.00 \\
& 3 & 61 & 46 & 75.41 \\
& 4 & 100 & 70 & 70.00 \\
& 5 & 61 & 0 & 0.00 \\
& 6 & 5 & 5 & 100.00 \\
Herd sizes & 7 & 61 & 60 & 98.36 \\
(animal) & $<1000$ & 127 & 65 & 51.18 \\
& $\geq 600-<6000$ & 183 & 167 & 91.26 \\
\multirow{2}{*}{ Time of vaccination } & $30-\leq 40$ & 100 & 70 & 70.00 \\
\hline (days) & $40-\leq 60$ & 127 & 66 & 51.96 \\
& $>60-\leq 90$ & 122 & 120 & 98.36 \\
& Sow & 161 & 116 & 72.04 \\
\hline \multirow{2}{*}{ Type of pigs } & Gilt & 71 & 52 & 73.24 \\
& Boar & 64 & 43 & 67.19 \\
& Grower & 60 & 32 & 53.33 \\
& & 215 & 175 & 81.40 \\
\hline
\end{tabular}

observed in pigs after vaccination $(P<0.05)$ in this study. In North Central provinces, Bui (2001) reported that the proportions of positive pigs for antibodies against CSFV after 21 days, 3 months and 6 months post-vaccination were $84.66 \%, 78.88 \%$, and $35.15 \%$, respectively. Precausta et al. (1983); Terpstra et al. (1990) and Dahle and Liess (1995) reported that the vaccination with a C-strain CSF vaccine-induced neutralizing antibodies that usually appear about 2 weeks after vaccination and increase until at least 4-12 weeks; they can persist for many years after (a single) vaccination. However, Terpstra and Tielen (1976) also indicated that some pigs did not produce antibodies against CSFV after vaccination. Most of the sows that had been vaccinated once 1-3 years earlier did not respond with an increase in antibody titre upon a second vaccination (Terpstra \& Tielen, 1976). When pigs are vaccinated in the presence of maternal antibodies, the formation of neutralizing antibodies is markedly inhibited. However, when such pigs were vaccinated a second time, many animals did show a rise in antibody titre after the second vaccination (Terpstra \& Wensvoort, 1987). Likewise, Bui (2001) also confirmed that the appearance of virus-neutralizing serum antibodies could be inhibited by the presence of maternal antibodies in pigs being vaccinated against CSFV.

Furthermore, significant differences in sero- prevalence observed in vaccinated pigs were found among types of the pig $(P<0.05)$. In previous studies, the ratio of positive sows for antibodies against CSFV (70.97\%) was higher than that of growers $(53.62 \%)$ (Lam Hoang Kiet, 2009). Likewise, the seroprevalence of antibodies against CSFV in sows and growers was $89.05 \%$ and $52.21 \%$, respectively (Nguyen, 2013). Huynh (2018) reported that the proportion of positive sows for antibody against CSFV (91.33\%) was higher than that of growers $(78.80 \%)$.

Of 108 negative pigs for antibodies against CSFV tested to screen for CSFV-specific antigen by using IDEXX CSFV Ag Serum Plus Test, no pig was found to contain CSFV antigen. This result was consistent with the result of the Ho Chi Minh Department of Animal Health in $\mathrm{Cu}$ Chi district in 2018, of 180 serum samples were analyzed for the presence of CSF antigen, no pig was found to contain CSFV antigen (Department of Animal Health, 2018). However, future vaccine developments should be stronger focused on a tailored DIVA (differentiating infected from vaccinated animals) assay to identify the differentiation between infected and vaccinated animals.

\section{Conclusion}

In conclusion, of seven farms selected, the highest percentages of positive pigs for antibodies 
against CSFV in Farm 1 and Farm 2 of An Nhon Tay, Farm 6 of Hoa Phu and farm 7 of Trung Lap Thuong communes were over $95 \%$ and no positive pigs in Farm 5 of Phu My Hung commune. Significant differences about the seroprevalence were observed in vaccinated pigs across herd size, days post-vaccination, type of pigs. No pig was found to contain CSFV antigen.

\section{References}

Bui, A. Q. (2001). Epidemiology of swine fever and prevention measures in some provinces of North Central of Vietnam (Unpublished doctoral dissertation). National Veterinary Institute, Ha Noi, Vietnam.

Huang Y. L., Deng M. C., Wang F. I., Huang C. C., \& Chang C. Y. (2014). The challenges of classical swine fever control: modified live and E2 subunit vaccines. Virus Research 179, 1-11.

Huynh, H. T. (2018). Circulation of virus and antibodies to swine fever in $\mathrm{Cu} C \mathrm{Chi}$ and Hoc Mon districts (Unpublished master's thesis). Nong Lam University, Ho Chi Minh City, Vietnam.

Lam, K. H. (2009). Survey the rate of swine cholera virus infection and effectiveness of protection after vaccination against swine fever virus in $\mathrm{Cu}$ Chi district (Unpublished bachelor's thesis). Nong Lam University, Ho Chi Minh City, Vietnam.

Meuwissen M. P. M., Horst, H. S., Huirne, R. B. M., \& Dijkhuizen, A. A. (1999). A model to estimate the financial consequences of classical swine fever outbreaks: principals and outcomes. Preventive Veterinary Medicine 42, 249-270.
MARD (Ministry of Agriculture and Rural Development). (2016). "The decision No. 07/2016/TT-TTBNNPTNT on prevention and control of terrestrial animal diseases" issued on 31 May, 2016. Ha Noi, Vietnam: MARD Office.

Nguyen, T. L. (2018). Survey the rate of swine cholera virus infection and effectiveness of protection after vaccination against swine fever virus in $\mathrm{Cu} C \mathrm{Chi}$ district (Unpublished bachelor's thesis). Nong Lam University, Ho Chi Minh City, Vietnam.

Nguyen, H. T. (2013). Seroprevalence against classical swine fever virus in vaccinated pigs and the circulation of virus in Binh Chanh, $\mathrm{Cu}$ Chi and Hoc Mon districts (Unpublished bachelor's thesis). Nong Lam University, Ho Chi Minh City, Vietnam.

Ratundima, E. M., Suartha, I. N., \& Mahardika, I. G. N. K. (2012). Deteksi antibodi terhadap virus classical swine fever dengan Teknik enzyme-linked immunosorbent assay. Indonesia Medicus Veterinus 1(2), 217-227.

Suradhat, S., Damrongwatanapokin, S., \& Thanawongnuwech, R. (2007). FactorsCritical for successful vaccination against classical swine fever in Endemic areas. Veterinary Microbiology 119, 1-9.

Terpstra, C., \& Wensvoort, G. (1987). Influence of the vaccination regime on the herd immune response for swine fever. Veterinary Microbiology 13(2), 143-151.

Terpstra, C., \& Tielen, M. J. M.(1976). Antibody response against swine fever following vaccination with the C-strain virus. Zentralblatt für Veterinärmedizin Reihe 23, 809-821.

WAHID (World Animal Health Information Database). (2015). Classical swine fever distribution map. Retrieved March 30, 2020, from https://www.oie.int/fr/. 